\title{
Assessing the accuracy of the isotropic periodic sum method through Madelung energy computation
}

\author{
Pedro Ojeda-May and Jingzhi Pua) \\ Department of Chemistry and Chemical Biology, Indiana University-Purdue University Indianapolis, \\ 402 N. Blackford Street, LD326, Indianapolis, Indiana 46202, USA
}

(Received 12 January 2014; accepted 8 April 2014; published online 25 April 2014)

\begin{abstract}
We tested the isotropic periodic sum (IPS) method for computing Madelung energies of ionic crystals. The performance of the method, both in its nonpolar (IPSn) and polar (IPSp) forms, was compared with that of the zero-charge and Wolf potentials [D. Wolf, P. Keblinski, S. R. Phillpot, and J. Eggebrecht, J. Chem. Phys. 110, 8254 (1999)]. The results show that the IPSn and IPSp methods converge the Madelung energy to its reference value with an average deviation of $\sim 10^{-4}$ and $\sim 10^{-7}$ energy units, respectively, for a cutoff range of $18-24 a$ ( $a / 2$ being the nearest-neighbor ion separation). However, minor oscillations were detected for the IPS methods when deviations of the computed Madelung energies were plotted on a logarithmic scale as a function of the cutoff distance. To remove such oscillations, we introduced a modified IPSn potential in which both the localregion and long-range electrostatic terms are damped, in analogy to the Wolf potential. With the damped-IPSn potential, a smoother convergence was achieved. In addition, we observed a better agreement between the damped-IPSn and IPSp methods, which suggests that damping the IPSn potential is in effect similar to adding a screening potential in IPSp. (C) 2014 AIP Publishing LLC. [http://dx.doi.org/10.1063/1.4871871]
\end{abstract}

\section{INTRODUCTION}

Computation of electrostatic energies has remained one of the most challenging tasks in condensed-phase simulations because of the long-range nature of the interactions involved. ${ }^{1}$ A reference problem for condensed-phase systems where electrostatics plays a crucial role is the computation of the Madelung energy for ionic crystals. ${ }^{2-6}$ This problem was first addressed by Madelung ${ }^{2}$ and later solved exactly by Ewald. ${ }^{3}$ In the Madelung problem, the energy is evaluated by adding the Coulombic, $r^{-1}$, term over all ion pairs. Lattice methods such as Ewald summation ${ }^{3}$ are the standard approaches to compute Madelung energies and electrostatic interactions in general. Although working well for many systems, they have been argued in some cases to cause artifacts because of the imposed periodicity, ${ }^{7,8}$ especially when nonuniform or non-neutral systems are concerned ${ }^{9}$ (see Ref. 1 for updates and alternative views). In addition to this, for largesized systems the best scaling behavior of modern Ewald methods, such as the particle-mesh-Ewald (PME) method, ${ }^{10}$ is $N \log (N)$ ( $N$ being the number of particles). The simple cutoff method is computationally more efficient as it scales linearly with $N$. However, when applied to the Madelung problem, it displays an unphysical oscillating behavior along the cutoff size due to the varying charge state of the cutoff sphere ${ }^{6}$ and thus is generally not recommended. ${ }^{11-13}$

Recently, substantial efforts have been put forward to develop reliable electrostatic treatments that take advantage of the efficiency of the cutoff-based schemes with an aim to remove the charge-dependent artifacts. ${ }^{6,14-17}$ This is achieved

\footnotetext{
a)Electronic mail: jpu@iupui.edu
}

in some methods by using a counter-charge term, which in effect makes the cutoff sphere neutral. Examples of this kind include, the simple zero-charge (ZC) method ${ }^{6,18}$ wherein a single correction term is added to the normal cutoff summation and the Wolf method ${ }^{6}$ wherein an additional damping factor is further applied. Both methods have been demonstrated effective in computing Madelung energies. Nevertheless, longrange electrostatic effects are not explicitly included in both these methods.

Following a philosophy different from both lattice summation and charge neutralization, $\mathrm{Wu}$ and Brooks ${ }^{19-21}$ introduced a linear-scaling method called the isotropic periodic sum (IPS) method, which includes a long-range correction term computed on the basis of homogeneity of a system. Here, a region of radius $R_{\mathrm{c}}$, called the local region, is employed to construct the isotropic charge distributions in distant regions of the system. The long-range electrostatic contributions are then evaluated over these virtual "copies" of the local region in a mean-field manner. Thus, the use of periodic lattice images is avoided. Wu and Brooks proposed two different versions of the IPS potentials: the nonpolar IPS potential (IPSn) ${ }^{19}$ which deals with charge-charge interactions and the polar IPS potential (IPSp) ${ }^{21}$ which is more suitable for systems involving charge-dipole and dipole-dipole interactions. Takahashi et al. further validated and refined the IPS methods for classical simulations. ${ }^{22,23}$ Recently, the IPS method has been successfully extended by us to the combined quantum mechanical and molecular mechanical (QM/MM) framework, with which good performance comparable to that of the QM/MM-Ewald method was observed. ${ }^{24}$ Despite these documented successes, the benchmark of the IPS method on the classic Madelung problem has not yet been established. 
In the present work, we assess the accuracy of the IPS method, both in its IPSn and IPSp forms, by obtaining the Madelung energy for the $\mathrm{NaCl}$ crystal and comparing the results with those of the ZC and Wolf potentials. In addition, a damped IPSn potential is obtained, which removes the minor energy oscillations detected in the original IPSn calculations.

\section{THEORY}

In what follows we will use the set $\mathcal{S}_{R_{\mathrm{c}}}[i]=\left\{q_{j}: j\right.$ $\left.\in \mathbb{N} \mid 0 \leq r_{i j} \leq R_{\mathrm{c}}\right\}$ which contains the charges $q_{j}$ located at a distance $\leq R_{\mathrm{c}}$ from a central charge $q_{i}$ (including $q_{i}$ ). In analogy to this, we define the set $\mathcal{S}_{R_{\mathrm{c}}}[i]-\{i\}$ which excludes the charged particle $i$.

The simple charge neutralization scheme leads to the $\mathrm{ZC}$ potential computed according to Ref. 6:

$$
E_{i}^{\mathrm{ZC}}=\sum_{j \in \mathcal{S}_{R_{\mathrm{c}}[i]-\{i\}}} \frac{q_{i} q_{j}}{r_{i j}}-\frac{q_{i}}{R_{\mathrm{c}}} \sum_{j \in \mathcal{S}_{R_{\mathrm{c}}[i]}} q_{j} .
$$

It was observed previously, that although this potential gives approximate values for the Madelung energy of crystals, it shows noticeable oscillations that decrease slowly as the cutoff radius increases. These oscillations can be damped by multiplying the Coulombic term by an $\operatorname{erfc}(\alpha r)$ factor, with $\alpha$ being a control parameter. The resulting potential is called the Wolf potential (see Eq. (5.13) in Ref. 6), which can be written as

$$
\begin{aligned}
E_{i}^{\text {Wolf }}= & q_{i} \sum_{j \in \mathcal{S}_{R_{\mathrm{c}}}[i]-\{i\}}\left[\frac{q_{j}}{r_{i j}} \operatorname{erfc}\left(\alpha r_{i j}\right)-\frac{q_{j}}{R_{\mathrm{c}}} \operatorname{erfc}\left(\alpha R_{\mathrm{c}}\right)\right] \\
& -\left[\frac{1}{R_{\mathrm{c}}} \operatorname{erfc}\left(\alpha R_{\mathrm{c}}\right)+\frac{2 \alpha}{\pi^{1 / 2}}\right] q_{i}^{2} .
\end{aligned}
$$

In the IPS method, the long-range-included pairwise electrostatic energy can be written as ${ }^{19}$

$$
\epsilon_{i j}^{\mathrm{IPS}}\left(r_{i j}\right)=\left\{\begin{array}{ll}
\epsilon_{i j}\left(r_{i j}\right)+\phi_{i j}\left(r_{i j}\right) & \text { if } r_{i j} \leq R_{\mathrm{c}} \\
0 & \text { otherwise }
\end{array},\right.
$$

where $\epsilon_{i j}$ is the Coulombic term and $\phi_{i j}$ is the long-range IPS correction whose operational expression is given by

$$
\phi_{i j}\left(r_{i j}\right)=\frac{q_{i} q_{j}}{R_{\mathrm{c}}}\left[\sum_{k=1}^{6} b_{2 k}\left(\frac{r_{i j}}{R_{\mathrm{c}}}\right)^{2 k}\right] .
$$

Note that in Eq. (4) the explicit dependence of $\epsilon_{i j}^{\mathrm{IPS}}, \epsilon_{i j}$, and $\phi_{i j}$ on $R_{\mathrm{c}}$ has been omitted for brevity.

Two different versions of IPS potentials called IPS nonpolar (IPSn) and IPS polar (IPSp) have been previously introduced. ${ }^{19,21}$ The former deals with ionic systems and the latter is intended for systems where charge-dipole and dipoledipole interactions are important. Treatment of such interactions in the IPSp method is made possible through a screening potential $\phi_{\text {screen }}$ which attenuates the field strength of the IPSn potential at large distances. In practice, the difference between IPSn and IPSp is reflected in the numerical values of the expansion coefficients which are given in Table I for both.

As pointed out by Wu and Brooks, the total IPS energy across the boundary of the local region would not be a con-
TABLE I. Coefficients for the IPSn (Ref. 19) and IPSp (Ref. 21) potentials.

\begin{tabular}{lcr}
\hline \hline Coefficient & IPSn & IPSp \\
\hline$b_{2}$ & 0.3018373 & 2.1875 \\
$b_{4}$ & 0.0648044 & -1.3125 \\
$b_{6}$ & 0.0157855 & 0.3125 \\
$b_{8}$ & 0.0037972 & $\ldots$ \\
$b_{10}$ & 0.0012149 & $\ldots$ \\
$b_{12}$ & -0.0000110 & $\cdots$ \\
\hline \hline
\end{tabular}

tinuous function if left untreated. ${ }^{19}$ Therefore, they suggested the use of the following pairwise interaction term, referred to as the configurational interaction (labeled with a superscript "C"), instead of Eq. (3):

$$
\epsilon_{i j}^{\mathrm{C}}\left(r_{i j}\right)=\left\{\begin{array}{ll}
-\epsilon_{i i}^{\mathrm{IPS}}\left(R_{\mathrm{c}}\right) & 1-1 \text { term } \\
\epsilon_{i j}^{\mathrm{IPS}}\left(r_{i j}\right)-\epsilon_{i j}^{\mathrm{IPS}}\left(R_{\mathrm{c}}\right) & \text { otherwise }
\end{array},\right.
$$

where the 1-1 term arises from the self interaction associated with the boundary treatment. Note that the present system does not contain 1-2,1-3, and 1-4 pairs, therefore these bonded interactions are not discussed. In this way, the total energy of the system is obtained as

$$
E^{\mathrm{IPS}}=\frac{1}{2} \sum_{i}^{N} \sum_{j}^{N} \epsilon_{i j}^{\mathrm{C}}+E^{\mathrm{B}},
$$

where $E^{\mathrm{B}}$ is the so-called boundary energy. ${ }^{19}$ For periodic systems, the boundary energy is expressed as ${ }^{19,21}$

$$
E^{\mathrm{B}}=\frac{V_{0}}{V} \epsilon^{\mathrm{IPS}}\left(R_{\mathrm{c}}\right)\left(\sum_{i}^{N} q_{i}\right)^{2},
$$

where $V_{0}$ and $V$ denote the volume of the local region and that of the periodic boundary box, respectively; $\epsilon^{\mathrm{IPS}}$ is the chargeindependent kernel of Eq. (3). It is important to note that the boundary energy term can be neglected here because the primary cell system can be extended to give an arbitrarily large volume $(V \rightarrow \infty)$ without affecting the computed values of Madelung energy per ion. Another way to justify the omission of boundary energy in the present study is that one can always construct a primary cell system for which the sum of the charges in Eq. (7) becomes zero. Thus, the total energy of particle $i$ can be written as

$$
E_{i}^{\mathrm{IPS}}=\sum_{j \in \mathcal{S}_{R_{\mathrm{c}}[i]-\{i\}}} \epsilon_{i j}^{\mathrm{C}}\left(r_{i j}\right)+\epsilon_{i i}^{\mathrm{C}}\left(R_{\mathrm{c}}\right),
$$

where the second term on right arises from the 1-1 term. Note that with this choice of boundary and configurational energies, no additional charge correction is needed for the IPS calculations, in contrast to the $\mathrm{ZC}$ and Wolf treatments.

\section{RESULTS AND DISCUSSION}

To test these existing methods, we computed the Madelung energy per ion for a periodically repeated $\mathrm{NaCl}$ crystal system using the ZC (Eq. (1)), Wolf (Eq. (2)), and both IPS potentials (Eq. (8)). In practice, this is equivalent to computing the electrostatic energy for particle $i$ located at 


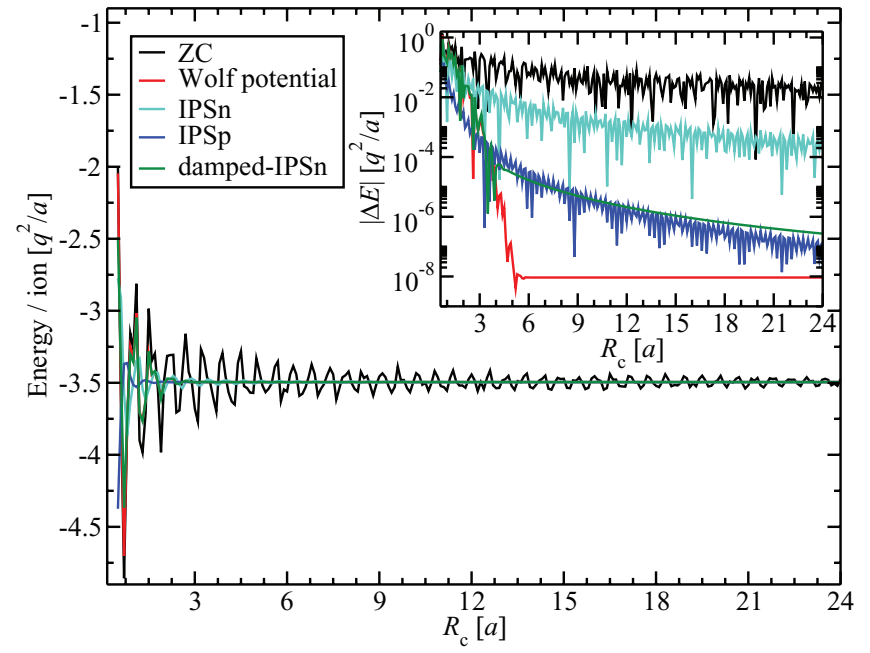

FIG. 1. Energy per ion computed from the ZC, Wolf, and three IPS potentials (IPSn, IPSp, and damped-IPSn). Inset: deviations with respect to the reference Madelung energy plotted on a log scale.

the center of a cubic block of the crystal. In what follows, we assume a box length of $l>2 R_{\mathrm{c}}$ and a nearest-neighbor ion separation of $a / 2$. In the Wolf potential calculations, we used a value of $\alpha=0.8 / a$ for the control parameter. ${ }^{25}$ The computed Madelung energies are shown in Fig. 1 as a function of the cutoff distance. Although all four methods, including IPSn and IPSp, tend to converge to the reference Madelung energy at large cutoff distances, the ZC method shows substantial oscillations whose amplitude decreases as the cutoff radius increases. The Wolf potential, IPSn, and IPSp methods display a much lower degree of oscillations compared to the $\mathrm{ZC}$ method such that the convergence curves of the three methods cannot be easily distinguished from one another for $R_{\mathrm{c}}>6 a$.

To monitor the detailed convergence behavior of these methods, we also calculated the deviations of the computed Madelung energies from its reference value $E_{\mathrm{Mad}}$ as follows:

$$
|\Delta E|=\left|E_{i}^{X}-E_{\mathrm{Mad}}\right|,
$$

where $X$ refers to any of the methods discussed above and $E_{\mathrm{Mad}}$ is the Madelung constant which is equal to $-3.49512918 q^{2} / a$ (see Ref. 6). The energy deviations for all methods are plotted in the inset of Fig. 1 on a log scale. We can see that the ZC method displays the largest deviation which is on average $\sim 10^{-2}$ energy units over the $R_{\mathrm{c}}$ range of $18-24 a$. The Wolf method seems to converge for the cutoff distances $R_{\mathrm{c}}>6 a$ with a deviation of $10^{-8}$ energy units. The IPSn method deviates from the reference value by $\sim 10^{-4}$ energy units for the $R_{\mathrm{c}}$ range of $18-24 a$, whereas the IPSp method results in an average deviation of $\sim 10^{-7}$ energy units for the same range of cutoff radius.

Large energy oscillations in the ZC calculations can be clearly seen from the corresponding deviation curve. Interestingly, the Wolf method does not show significant oscillations for $R_{\mathrm{c}}>6 a$. Although the IPSn and IPSp methods display a deceptively similar oscillating pattern as that observed in the ZC method on a logarithmic scale, the actual oscillations in the IPS methods are much smaller than those in the ZC method. For example, the oscillations in the IPSn and IPSp methods are bounded by $\sim 10^{-4}$ and $\sim 10^{-7}$ energy units over the $R_{\mathrm{c}}$ range of $18-24 a$, whereas the oscillations in the $\mathrm{ZC}$ method for the same cutoff range are in the order of $\sim 10^{-2}$ energy units. Recall that the IPSp method differs from the IPSn method by a screening potential contribution. Thus, the addition of the screening potential to IPSn seems to decrease its level of oscillations, making the IPSp method to converge the Madelung energy more rapidly and more smoothly. However, even when the oscillations are reduced considerably, they are not completely avoided in the two IPS methods.

To remove the oscillations at large cutoff distances in the IPSn method, we have studied a damped IPSn potential (designated damped-IPSn), which is given by

$$
\begin{aligned}
E_{i}^{\text {damped-IPSn }}= & \sum_{j \in \mathcal{S}_{R_{\mathrm{c}}}[i]-\{i\}} \epsilon_{i j}^{\text {damped-C }}\left(r_{i j}\right) \\
& +\epsilon_{i i}^{\text {damped-C }}\left(R_{\mathrm{c}}\right)-\frac{2 \alpha}{\pi^{1 / 2}} q_{i}^{2},
\end{aligned}
$$

where the first two terms on right correspond to the damped IPS interactions in Eq. (8). Although the so-called self term (the last term on the right-hand side of Eq. (10)) cannot be deduced directly from the original set of the IPS equations in Ref. 19, it can be obtained by following a similar procedure used by Wolf et al. ${ }^{6}$ for charge neutralization. We found inclusion of this self term essential for the dampedIPSn method to converge the Madelung energy. The expression of the $\epsilon_{i j}^{\text {damped-C }}$ term is given by

$$
\begin{aligned}
& \epsilon_{i j}^{\text {damped-C }} \\
& \quad= \begin{cases}-\epsilon_{i i}^{\mathrm{IPS}}\left(R_{\mathrm{c}}\right) \operatorname{erfc}\left(\alpha R_{\mathrm{c}}\right) & 1-1 \text { term } \\
\epsilon_{i j}^{\mathrm{IPS}}\left(r_{i j}\right) \operatorname{erfc}\left(\alpha r_{i j}\right)-\epsilon_{i j}^{\mathrm{IPS}}\left(R_{\mathrm{c}}\right) \operatorname{erfc}\left(\alpha R_{\mathrm{c}}\right) & \text { otherwise }\end{cases}
\end{aligned}
$$

Note that Eq. (10) resembles the form of the Wolf potential in Eq. (2) except that the $r^{-1}$ term is substituted by the IPS potential.

We plotted the energy per ion obtained by the dampedIPSn method (with $\alpha=0.8 / a)^{25}$ in Fig. 1 together with the deviation from the reference Madelung energy, which is shown in the inset panel. The damped-IPSn method converges to the reference Madelung energy with an average deviation of $\sim 10^{-7}$ energy units over the cutoff range of $18-24 a$. Other two important features can be noticed from the curve of the damped-IPSn method: first, the oscillations are removed; second, the damping factor turns the original IPSn curve into the envelop curve of the IPSp curve. These results suggest that in addition to facilitating convergence of the IPSn method, the damping factor may also act similarly as the screening potential present in the IPSp method.

In conclusion, the IPSn and IPSp methods accurately converge the computed Madelung energy to its reference value with an uncertainty of $\sim 10^{-4}$ and $\sim 10^{-7}$ energy units, respectively, which is similar to that of the Wolf method ( $\sim 10^{-8}$ energy units, or specifically $\sim 2 \times 10^{-6} \mathrm{kcal} / \mathrm{mol}$ for $\mathrm{NaCl}$ ). This level of agreement is encouraging considering that electrostatics is handled quite differently in these 
methods. We also found that the screening potential included in the IPSp method may serve a similar function of the damping term, which decreases the level of oscillations in IPSn.

\section{ACKNOWLEDGMENTS}

We thank Dr. Xiongwu Wu and Dr. Bernard Brooks for insightful discussions. This work was supported by a start-up grant from Indiana University-Purdue University Indianapolis (IUPUI). Computing time was provided through a HighPerformance Computing Cluster funded by the School of Science at IUPUI.

${ }^{1}$ G. A. Cisneros, M. Karttunen, P. Ren, and C. Sagui, Chem. Rev. 114, 779 (2014).

${ }^{2}$ E. Madelung, Phys. Z. XIX, 524 (1918).

${ }^{3}$ P. P. Ewald, Ann. Phys. (Leipzig) 369, 253 (1921).

${ }^{4}$ W. A. Harrison, Phys. Rev. B 73, 212103 (2006).

${ }^{5}$ M. Gaio and P. L. Silvestrelli, Phys. Rev. B 79, 012102 (2009).

${ }^{6}$ D. Wolf, P. Keblinski, S. R. Phillpot, and J. Eggebrecht, J. Chem. Phys. 110, 8254 (1999).

${ }^{7}$ B. A. Luty, I. G. Tironi, and W. F. van Gunsteren, J. Chem. Phys. 103, 3014 (1995).
${ }^{8}$ Z. Kurtović, M. Marchi, and D. Chandler, Mol. Phys. 78, 1155 (1993).

${ }^{9}$ J. S. Hub, B. L. de Groot, H. Grubmüller, and G. Groenhof, J. Chem. Theory Comput. 10, 381 (2014).

${ }^{10}$ T. Darden, D. York, and L. Pedersen, J. Chem. Phys. 98, 10089 (1993).

${ }^{11}$ J. S. Bader and D. Chandler, J. Phys. Chem. 96, 6423 (1992).

${ }^{12}$ P. E. Smith and B. M. Pettitt, J. Chem. Phys. 95, 8430 (1991).

${ }^{13}$ C. L. Brooks III, B. M. Pettitt, and M. Karplus, J. Chem. Phys. 83, 5897 (1985).

${ }^{14}$ D. Zahn, B. Schilling, and S. M. Kast, J. Phys. Chem. B 106, 10725 (2002).

${ }^{15}$ C. J. Fennell and J. D. Gezelter, J. Chem. Phys. 124, 234104 (2006).

${ }^{16}$ Y. Yonezawa, J. Chem. Phys. 136, 244103 (2012).

${ }^{17}$ B. W. McCann and O. Acevedo, J. Chem. Theory Comput. 9, 944 (2013).

${ }^{18}$ I. Fukuda, Y. Yonezawa, and H. Nakamura, J. Phys. Soc. Japan 77, 114301 (2008).

${ }^{19} \mathrm{X} . \mathrm{Wu}$ and B. R. Brooks, J. Chem. Phys. 122, 044107 (2005).

${ }^{20} \mathrm{X}$. Wu and B. R. Brooks, J. Chem. Phys. 129, 154115 (2008).

${ }^{21} \mathrm{X} . \mathrm{Wu}$ and B. R. Brooks, J. Chem. Phys. 131, 024107 (2009).

${ }^{22}$ K. Z. Takahashi, T. Narumi, and K. Yasuoka, J. Chem. Phys. 135, 174108 (2011).

${ }^{23}$ K. Z. Takahashi, T. Narumi, D. Suh, and K. Yasuoka, J. Chem. Theory Comput. 8, 4503 (2012).

${ }^{24}$ P. Ojeda-May and J. Pu, J. Chem. Theory Comput. 10, 134 (2014).

${ }^{25} \mathrm{See}$ supplementary material at http://dx.doi.org/10.1063/1.4871871 for choice of the control parameter. 